\title{
Performance Analysis of a Wireless Network with Opportunistic Spectrum Sharing
}

\author{
Shensheng Tang and Brian L. Mark \\ Dept. of Electrical and Computer Engineering \\ George Mason University, Fairfax, VA
}

\begin{abstract}
We analyze the performance of a wireless system that allows opportunistic spectrum sharing. The system consists of a set of primary users sharing a set of channels over a coverage area. The resources allocated to the primary users are shared opportunistically with a set of secondary users. The secondary users are capable of detecting channels that are unused by the primary users and then making use of the idle channels. If no channel is available for a secondary call, the call waits in a buffer until either a channel becomes available or a maximum waiting time is reached. We compute the blocking probabilities, mean reconnection probability, channel utilization, and total carried traffic in the system. Our results suggest that opportunistic spectrum sharing can significantly improve the efficiency of a wireless system, without negatively impacting the performance seen by the primary users.
\end{abstract}

\section{INTRODUCTION}

Studies of wireless spectrum usage [1], [2] have shown that large portions of the allocated spectrum are highly underutilized. Frequency agile radios (FARs) are cognitive radios that are capable of detecting idle frequency channels and opportunistically making use of them without causing harmful interference to the primary users [3]. In such a scenario of opportunistic spectrum sharing (OSS), the FARs are called secondary users and the owners of the allocated spectrum are the primary users. By allowing secondary users to reclaim idle channels, a much higher spectrum efficiency can be achieved, in principle. More generally, cognitive radios [4] may be capable of opportunistic spectrum access over frequency channels, time slots, or spreading codes.

In this paper, we evaluate the performance of a generic opportunistic spectrum sharing (OSS) system in terms of blocking probability, reconnection probability, channel utilization, and total carried traffic. We consider a wireless network, which provides a group of channels to a set of primary users. The wireless network may or may not have an infrastructure. Here, we use the term channel in a broad sense. A channel could be a frequency channel in an FDMA system, a time-slot in a TDMA system, a spreading code in a CDMA system, or a tone in an OFDM system. Our system model can be applied to all of these scenarios.

Secondary users opportunistically make use of channels that are not occupied by primary users. We assume that the secondary users are capable of sensing when a channel is idle and then making use of such a channel. Conversely, a secondary user can detect when a primary user accesses a channel that it is using and then either move to another channel, if an idle channel is available, or move to a waiting pool. In the latter case, the secondary user's call waits in a buffer until either a new channel becomes available or until a timeout occurs after a predefined maximum waiting time.

The reliable detection of primary users is a major challenge for the implementation of an OSS system. The spectrum usage of the secondary users is contingent on the requirement that the interference to the primary users must be limited to a certain threshold. A number of opportunistic spectrum access (OSA) schemes have been developed recently in the literature [3], [5]-[7]. This paper focuses on the performance analysis of an OSS system model at the call level under the assumption of perfect OSA, i.e., the secondary users are able to move in and out of channels to avoid harmful interference with primary users.

The remainder of the paper is organized as follows. Section II describes the system model and assumptions in further detail. Section III develops a Markovian model of the system dynamics and derives the performance metrics of interest. Section IV presents numerical results, illustrating the performance of the OSS system with respect to the different metrics over a range of parameter settings. Finally, the paper is concluded in Section V.

\section{Model AND Assumptions}

The OSS system includes two types of wireless networks operating over a given service area. The network that owns the license for spectrum usage in the service area is referred to as the primary system. The users of this network are the primary users. Calls generated by primary users constitute the primary traffic (PT) stream. The other network in the same service area, which opportunistically shares the precious spectrum resource with the primary system, is referred to as the secondary system and the associated users are called secondary users. Calls generated by secondary users constitute the secondary traffic (ST) stream.

In the OSS system, the spectrum availability for the secondary users depends on the spectrum occupancy of the primary users. A distinct feature of the OSS system is that the secondary users have the capability to sense channel usage and switch between different channels using appropriate communication mechanisms, while causing negligible interference to the primary 


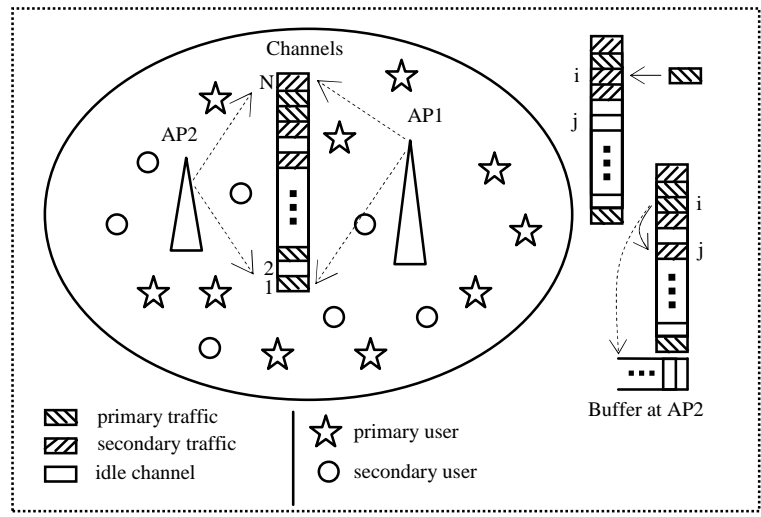

Fig. 1. The opportunistic spectrum sharing (OSS) system model.

users. Such functionality can be realized by frequency agile radios. As an example, FARs have the ability to transmit and receive signals on dynamically tunable frequency ranges and can employ various spectrum access mechanisms, such as the Listen-Before-Talk scheme [3].

An OSS system model is depicted in Fig. 1. In this system model, we assume that the primary system and secondary system are both infrastructured wireless networks. However, the performance model discussed in this paper applies to more general scenarios. For example, one or both of the primary and secondary systems may be infrastureless ad hoc networks. Suppose there are a total of $N$ channels managed by the primary system with access point AP1 in a given cell. The PT calls operate as if there are no ST calls in the system. When a PT call arrives to the system, it occupies a free channel if one is available; otherwise, it will be blocked. Secondary users detect the presence or absence of signals from primary users and maintain records of the channel occupancy status. The detection mechanism may involve collaboration with other secondary users and/or an exchange with an associated access point called AP2, as shown in Fig. 1.

When an ST node detects or is informed (by AP2 or other ST nodes) of an arrival of a PT call in its current channel, it immediately leaves the channel and switches to an idle channel, if one is available, to continue the call (see Fig. 1). If at that time all the channels are occupied, the ST call is placed into a buffer located at AP2. If the secondary system is infrastructureless, AP2 is not needed, but a virtual queue of ST calls would need to be maintained by the secondary users in a distributed manner. In Fig. 1, when an ST call detects the arrival of a PT call at channel $i$, it immediately leaves that channel and changes to channel $j$. If all of the $N$ channels are occupied at that time, the ST call will be queued. Queued ST calls are served in first-come first-served (FCFS) order. The head-of-line ST call is reconnected to the system when a channel becomes available before a predefined maximum tolerated waiting time (MTWT) expires. We set the MTWT of an ST call equal to its residence time in the considered service area. Thus, an ST call is lost if it is forced to wait for a period of time equivalent (in the statistical sense) to its residence time in the service area, i.e., until it moves out of the service area.

\section{Performance Analysis}

In this section, we analyze the OSS system performance in a given service area consisting of the primary and secondary systems sharing the same spectrum. The spectrum is divided into $N$ channels serving the two types of traffic: primary traffic (PT) and secondary traffic (ST). Arrivals of the PT and ST calls are assumed to form two independent Poisson processes with rates $\lambda_{1}$ and $\lambda_{2}$, respectively. The call holding times of the PT and ST calls are assumed to be exponentially distributed with means $1 / h_{1}$ and $1 / h_{2}$, respectively. The residence times for the PT and ST in the service area are also assumed to be exponentially distributed with means $1 / r_{1}$ and $1 / r_{2}$, respectively. The channel holding time is the minimum of the call holding and residence times. Hence, the channel holding times for the PT and ST calls are exponentially distributed with means $1 / \mu_{1}=1 /\left(h_{1}+r_{1}\right)$ and $1 / \mu_{2}=1 /\left(h_{2}+r_{2}\right)$, respectively. These assumptions have been widely used in literature [8]-[11] and have been found to be reasonable as long as the number of users is much more than that of the channels in a service area. We further assume that both types of traffic occupy one channel for simplicity. However, the analysis method used here can be extended to handle variable bandwidth requests (cf. [12]).

Let $X_{1}(t)$ denote the number of PT calls in the OSS system at time $t$. Similarly, let $X_{2}(t)$ be the number of ST calls in the system at time $t$, including the ST calls being served and those waiting in the buffer at AP2. The process $\left(X_{1}(t), X_{2}(t)\right)$ is a two-dimensional Markov process with state space

$$
\mathcal{S}=\left\{\left(n_{1}, n_{2}\right) \mid 0 \leq n_{1}, n_{2} \leq N\right\} .
$$

The transition rate from state $\left(n_{1}, n_{2}\right)$ to $\left(n_{1}^{\prime}, n_{2}^{\prime}\right)$, denoted by $T_{n_{1}, n_{2}}^{n_{1}^{\prime}, n_{2}^{\prime}}$, is given by

$$
\begin{aligned}
& T_{n_{1}, n_{2}}^{n_{1}+1, n_{2}}= \begin{cases}\lambda_{1}, & 0 \leq n_{1}<N, 0 \leq n_{2} \leq N, \\
0, & \text { otherwise. }\end{cases} \\
& T_{n_{1}, n_{2}}^{n_{1}-1, n_{2}}=n_{1} \mu_{1}, 1 \leq n_{1} \leq N, 0 \leq n_{2} \leq N . \\
& T_{n_{1}, n_{2}}^{n_{1}, n_{2}+1}=\left\{\begin{array}{cc}
\lambda_{2}, & 0 \leq n_{1} \leq N-1, \\
0, & 0 \leq n_{2}<N-n_{1},
\end{array}\right. \\
& T_{n_{1}, n_{2}}^{n_{1}, n_{2}-1}=\left\{\begin{array}{cc}
n_{2} \mu_{2}, & 0 \leq n_{1} \leq N-1, \\
\left(n_{2}-N+n_{1}\right) r_{2} & 1 \leq n_{2} \leq N-n_{1}, \\
+\left(N-n_{1}\right) \mu_{2}, & 1 \leq n_{1} \leq N, \\
& N-n_{1}<n_{2} \leq N .
\end{array}\right.
\end{aligned}
$$

Let $\pi\left(n_{1}, n_{2}\right)$ denote the steady-state probability that the OSS system is in state $\left(n_{1}, n_{2}\right)$. The steady-state system probability vector, with states ordered lexicographically, can be represented as

$$
\boldsymbol{\pi}=\left(\boldsymbol{\pi}_{0}, \boldsymbol{\pi}_{1}, \cdots, \boldsymbol{\pi}_{N}\right),
$$


where

$$
\boldsymbol{\pi}_{n}=(\pi(n, 0), \pi(n, 1), \cdots, \pi(n, N)), \quad 0 \leq n \leq N
$$

The vector $\pi$ is the solution of following equations:

$$
\boldsymbol{\pi} Q=\mathbf{0} \text { and } \boldsymbol{\pi} \mathbf{e}=\mathbf{1}
$$

where $\mathbf{e}$ and $\mathbf{0}$ are column vectors of all ones and zeros, respectively. The infinitesimal generator, $Q$, of the twodimensional Markov process, is given by

$Q=\left[\begin{array}{lllllll}E_{0} & B_{0} & 0 & \cdots & 0 & 0 & 0 \\ D_{1} & E_{1} & B_{1} & \cdots & 0 & 0 & 0 \\ \vdots & \vdots & \vdots & \vdots & \vdots & \vdots & \vdots \\ 0 & 0 & 0 & \cdots & D_{N-1} & E_{N-1} & B_{N-1} \\ 0 & 0 & 0 & \cdots & 0 & D_{N} & E_{N}\end{array}\right]$

where each submatrix is of size $N+1$ by $N+1$ and defined by

$$
\begin{aligned}
& B_{i}=\lambda_{1} I_{N+1}, \quad 0 \leq i<N \\
& D_{i}=i \mu_{1} I_{N+1}, \quad 1 \leq i \leq N \\
& E_{i}=A_{i}-\bar{\delta}(i) D_{i}-\bar{\delta}(N-i) B_{i}, \quad 0 \leq i \leq N,
\end{aligned}
$$

where $I_{n}$ denotes an $n$-by- $n$ identity matrix, $\bar{\delta}(i)$ is 0 when $i=0$ and 1 otherwise. Let $A_{i}(j, k)$ denote the $j$ th row and $k$-th column element of the matrix $A_{i}$, defined as follows:

$$
A_{i}(j, k)= \begin{cases}\lambda_{2}, & 0 \leq i \leq N-1, \\ j \mu_{2}, & 0 \leq j<N-i, k=j+1, \\ (N-i) \mu_{2} & 1 \leq j \leq N-i, k=j-1, \\ +(j-N+i) r_{2}, & 1 \leq i \leq N, \\ -\left[A_{i}(j, j-1)\right. & N-i<j \leq N, k=j-1, \\ \left.+A_{i}(j, j+1)\right], & 0 \leq i \leq N, \\ 0, & 0 \leq j \leq N, k=j, \\ 0 & \text { otherwise, }\end{cases}
$$

where $A_{i}(j, k) \triangleq 0$ for $j, k<0$ or $j, k>N$. Applying the method developed in [11], the equilibrium state probabilities can be determined as

$\boldsymbol{\pi}_{n}=\boldsymbol{\pi}_{n-1} B_{n-1}\left(-C_{n}\right)^{-1}=\boldsymbol{\pi}_{0} \prod_{i=1}^{n}\left[B_{i-1}\left(-C_{i}\right)^{-1}\right]$,

where $1 \leq n \leq N$ and $\boldsymbol{\pi}_{0}$ satisfies $\boldsymbol{\pi}_{0} C_{0}=\mathbf{0}$ and

$$
\boldsymbol{\pi}_{0}\left[I+\sum_{n=1}^{N} \prod_{i=1}^{n}\left[B_{i-1}\left(-C_{i}\right)^{-1}\right]\right] \mathbf{e}=1 \text {. }
$$

The $C_{i}$ are computed recursively as follows:

$$
\begin{aligned}
C_{N} & =E_{N} \\
C_{i} & =E_{i}+B_{i}\left(-C_{i+1}\right)^{-1} D_{i+1}, 0 \leq i \leq N-1 .
\end{aligned}
$$

After obtaining the steady state probabilities, it is straightforward to determine various performance measures of interest.

\section{A. Blocking probabilities}

- Blocking probability of the primary traffic The blocking probability of the PT, denoted by $P_{1}$, is defined as the probability that upon an arrival of a PT call in a service area all the channels are occupied by PT calls and the arrival request has to be blocked. Thus, we have

$$
P_{1}=\sum_{n_{2}=0}^{N} \pi\left(N, n_{2}\right)=\pi_{0} \prod_{i=1}^{N}\left[B_{i-1}\left(-C_{i}\right)^{-1}\right] \mathbf{e} .
$$

\section{- Blocking probability of the secondary traffic}

The blocking probability of the ST, denoted by $P_{2}$, is defined as the probability that upon an arrival of an ST call in a service area all the channels are occupied by either PT calls and/or ST calls and the arrival request has to be blocked. Thus, we have

$$
P_{2}=\sum_{n_{1}=0}^{N} \sum_{n_{2}=N-n_{1}}^{N} \pi\left(n_{1}, n_{2}\right) \text {. }
$$

\section{B. Mean reconnection probability}

As mentioned earlier, an ST call that waits in the buffer due to unavailability of a channel could reconnect back to the system if a channel becomes available before the maximum waiting time expires. The mean reconnection probability of an ST call, denoted by $\gamma$, is defined as the probability that this ST call reconnects back to the system before its MTWT expires. We can derive the expression of $\gamma$ as:

$$
\gamma=\frac{\sum_{n_{1}=1}^{N} \sum_{j=0}^{n_{1}-1} \pi\left(n_{1}, N-n_{1}+j+1\right) \beta(j)}{\sum_{n_{1}=1}^{N} \sum_{j=0}^{n_{1}-1} \pi\left(n_{1}, N-n_{1}+j+1\right)},
$$

where $\beta(j)$ denotes the conditional probability that an ST call arriving at the buffer eventually reconnects back to the system before its MTWT expires, given that the ST call comes to find that there are $j$ ST calls in the buffer $(0 \leq j \leq N-1)$.

We now proceed to derive an expression for $\beta(j)$. When an ST call detects the arrival of a PT call on its current channel, it switches to another idle channel if one is available; otherwise, the call is queued in the buffer at AP2. The ST calls in the buffer are reconnected to the system when channels become available in first come first served (FCFS) order. More precisely, if an ST call detects an arrival of a PT call at its channel and there are $N+j(0 \leq j \leq N-1)$ calls in the system (i.e., all $N$ channels are serving the PT/ST calls and $j$ ST calls are waiting in the buffer), it releases its channel for the PT call and enters the buffer, which leads to a new system state with all $N$ channels being used and $j+1$ ST calls in the buffer. This ST call reconnects to the system only if the $j+1$ calls leave the buffer (either by releasing a channel or by releasing a position in the buffer) before its MTWT expires.

Let $\tau$ denote the MTWT of an ST call in the buffer. As discussed earlier, the MTWT of an ST call in the buffer is assumed to be equivalent to the residence time 
of the ST call. Hence, $\tau$ is exponentially distributed with mean $E[\tau]=1 / r_{2}$. To capture the queueing behavior of ST calls, we introduce a process $J(t)$ to represent the number of queued ST calls at time $t$. Then the OSS system when all channels are occupied can be represented by a 3-dimensional Markov process $\left(Y_{1}(t), Y_{2}(t), J(t)\right)$ with state space

$$
\mathcal{S}^{*}=\left\{\left(n_{1}, n_{2}, j\right) \mid n_{1}+n_{2}=N, 0 \leq j \leq N\right\} .
$$

Let $\varphi_{j}(0 \leq j \leq N-1)$ denote the time interval, in steady-state, between a transition to a state $\left(n_{1}, N-\right.$ $\left.n_{1}, j+1\right) \in \mathcal{S}^{*}$ until a transition to a new state $\left(n_{1}^{\prime}, N-\right.$ $\left.n_{1}^{\prime}, j\right)$, such that either a PT/ST call occupying a channel leaves the system, or a queued ST call leaves the system. If a PT call leaves, then $n_{1}^{\prime}=n_{1}-1$; otherwise, $n_{1}^{\prime}=n_{1}$. When a PT or ST call leaves the system, the head-of-line ST call in the queue reconnects to the system and the remaining queued ST calls advance by one position in the buffer. Similarly, when a queued ST call is dropped, each of the remaining queued ST calls behind it advance by one position. Hence, $\varphi_{j}$ is exponentially distributed with parameter $g_{j}=n_{1} \mu_{1}+\left(N-n_{1}\right) \mu_{2}+j r_{2}, 0 \leq j \leq$ $N-1$.

Let $f_{j}(\cdot)$ denote the probability density function of $\varphi_{j}$ and let $f_{j}^{*}(s)$ denote the Laplace transform of $f_{j}(\cdot)$. By the independence assumption of the random variables $\varphi_{j}$, we can determine $\beta(j)$ as

$$
\begin{aligned}
\beta(j) & =\operatorname{Pr}\left(\tau>\varphi_{0}+\varphi_{1}+\cdots+\varphi_{j}\right)=\prod_{i=0}^{j} f_{i}^{*}\left(r_{2}\right) \\
& =\frac{n_{1} \mu_{1}+\left(N-n_{1}\right) \mu_{2}}{n_{1} \mu_{1}+\left(N-n_{1}\right) \mu_{2}+(j+1) r_{2}},
\end{aligned}
$$

where the last equation follows from the fact that

$$
f_{i}^{*}\left(r_{2}\right)=\frac{n_{1} \mu_{1}+\left(N-n_{1}\right) \mu_{2}+i r_{2}}{n_{1} \mu_{1}+\left(N-n_{1}\right) \mu_{2}+(i+1) r_{2}} .
$$

The reconnection probability can then be calculated by substituting (7) into (6).

\section{Total channel utilization}

The total channel utilization $\eta$ is defined as the ratio of the mean number of occupied channels to the total number of channels. We find that

$$
\begin{aligned}
\eta=\frac{1}{N} & \left\{\sum_{n_{1}=0}^{N} \sum_{n_{2}=0}^{N-n_{1}}\left(n_{1}+n_{2}\right) \pi\left(n_{1}, n_{2}\right)\right. \\
+ & \left.+\sum_{n_{1}=1}^{N} \sum_{n_{2}=N-n_{1}+1}^{N} N \pi\left(n_{1}, n_{2}\right)\right\} .
\end{aligned}
$$

\section{Total carried traffic}

The total carried traffic (TCT) by the OSS system is defined as the total traffic (both PT and ST) that the OSS system supports for conversation in the given service area. We find that

$$
\begin{aligned}
T C T & =\sum_{n_{1}=0}^{N} \sum_{n_{2}=0}^{N-n_{1}}\left(n_{1}+n_{2}\right) \pi\left(n_{1}, n_{2}\right) \\
& +\sum_{n_{1}=1}^{N} \sum_{n_{2}=N-n_{1}+1}^{N} N \pi\left(n_{1}, n_{2}\right) .
\end{aligned}
$$

\section{Numerical Results}

In this section, we present numerical results for the OSS system model under the following parameter settings:

$$
\mu_{1}=10, r_{1}=5, \mu_{2}=10, r_{2}=5 .
$$

Fig. 2 shows how the blocking probabilities in both systems change as the traffic intensities for both types of traffic are varied. We observe that both blocking probabilities increase as the PT intensity $\rho_{1} \triangleq \lambda_{1} / \mu_{1}$ increases. When the ST intensity $\rho_{2} \triangleq \lambda_{1} / \mu_{2}$ increases, only the ST call blocking probability increases. This is because in the OSS system, the channel availability for a ST call depends on the requirement that it cannot interfere with a PT call. On the other hand, the PT calls are oblivious to the existence of ST calls, under a perfect OSA mechanism. The latter can also be validated by comparison with the single primary system without ST calls, which actually operates as an M/M/N/N queuing system. As should be expected, the blocking probability for ST calls is higher than that of PT calls under the same parameter settings for both types of calls.

Fig. 3 shows the effect of the ST call residence time and the primary traffic intensity on queuing performance. We observe that the mean reconnection probability of an ST call decreases as $\rho_{1}$ increases, and increases as $E[\tau]$ increases. A higher volume of PT calls results in a smaller chance that a queued ST call reconnects to the system, while a longer maximum queueing time leads to a higher chance of reconnection.

In Fig. 4, we observe that the channel utilization increases with increasing $\rho_{1}$ and/or $\rho_{2}$ and the channel utilization of the OSS system is much higher than that of a single primary system. This is due to the fact that a given channel is occupied by a call regardless of whether it is of type PT or ST. Fig. 5 shows that the total carried traffic (TCT) has a similar performance trend with respect to channel utilization, i.e., the TCT increases increasing $\rho_{1}$ and/or $\rho_{2}$. In particular, TCT of the OSS system is much higher than that of a single primary system.

\section{CONCLUSION}

We presented a performance analysis of a wireless network with opportunistic spectrum sharing (OSS). Our analysis and numerical results show that OSS can significantly improve the spectrum efficiency and system capacity without negatively impacting the performance of the primary users. The analysis developed in this paper assumes that the secondary users are able to perfectly detect the presence or absence of a primary user on a channel. To make our model more realistic, the characteristics of an imperfect signal detection mechanism should be taken into account. This is a topic of ongoing work.

\section{ACKNOWLEDGMENT}

This work was supported in part by the U.S. National Science Foundation under Grant CNS-0520151. 


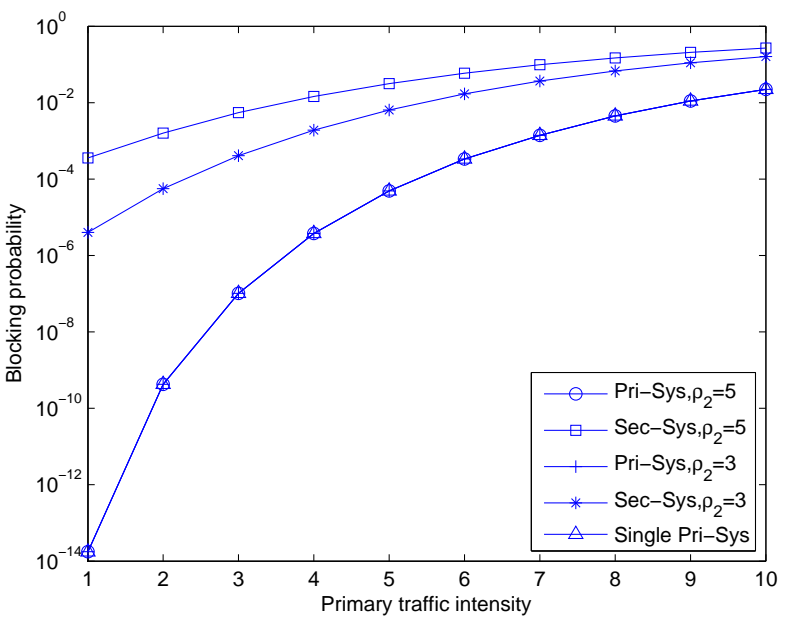

Fig. 2. Blocking probabilities vs. primary traffic intensity $\rho_{1}$.

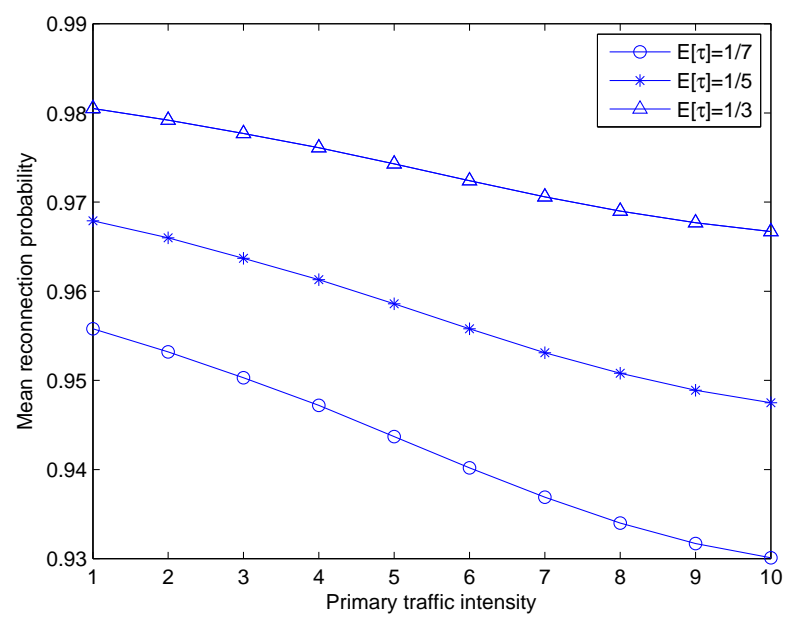

Fig. 3. Mean reconnection probability vs. $\rho_{1}$.

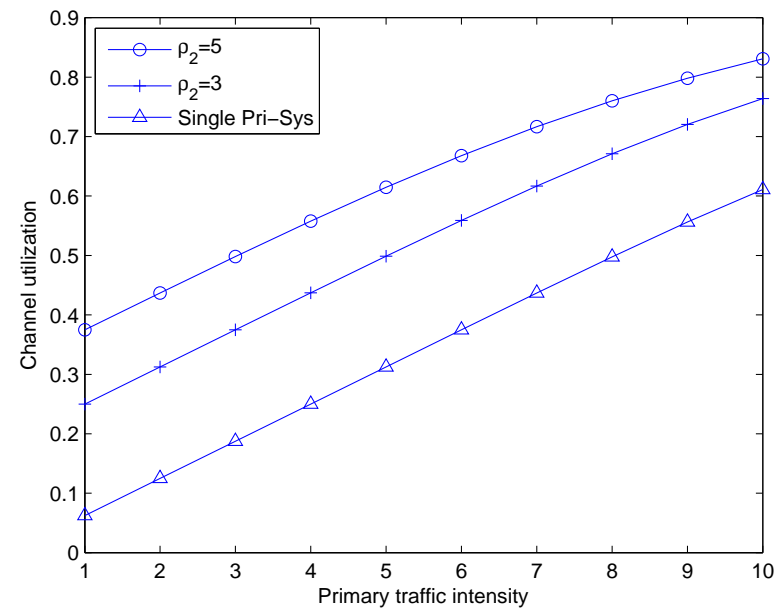

Fig. 4. Channel utilization vs. $\rho_{1}$.

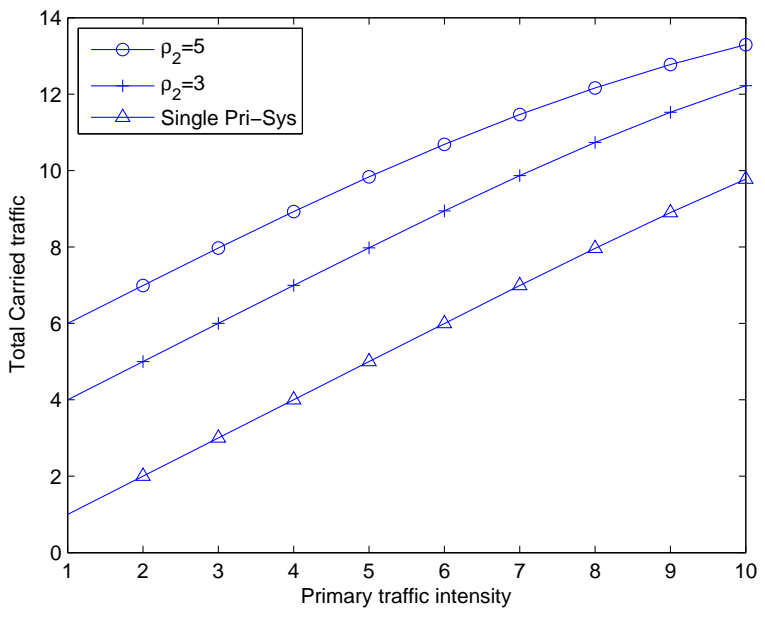

Fig. 5. Total carried traffic vs. $\rho_{1}$.

\section{REFERENCES}

[1] M. McHenry, "Frequency agile spectrum access technologies," in Proc. FCC Workshop on Cognitive Radio, May 2003.

[2] G. Staple and K. Werbach, "The end of spectrum scarcity," IEEE Spectrum, vol. 41, pp. 48-52, March 2004.

[3] A. E. Leu, M. McHenry, and B. L. Mark, "Modeling and analysis of interference in listen-before-talk spectrum access schemes," Int. J. Network Mgmt, vol. 16, pp. 131-147, 2006.

[4] S. Haykin, "Cognitive Radio: Brain-Empowered Wireless Communications," IEEE J. Selected Areas in Comm., vol. 23, pp. 201220, Feb. 2005.

[5] A. Ghasemi and E. Sousa, "Collaborative spectrum sensing for opportunistic access in fading environments," in Proc. IEEE Symp. on New Frontiers in Dynamic Spectrum Access Networks (DySPAN), pp. 131-136, Nov. 2005.

[6] B. Wild and K. Ramchandran, "Detecting primary receivers for cognitive radio applications," in Proc. IEEE Symp. on New Frontiers in Dynamic Spectrum Access Networks (DySPAN), pp. 124-130, Nov. 2005.

[7] S. Geirhofer, L. Tong, and B. Sadler, "A measurement-based model for dynamic spectrum access in wlan channels," in Proc. IEEE Military Commun. Conf. (Milcom), pp. 1-7, Oct. 2006.

[8] Y. Fang, Y.-B. Lin, and I. Chlamtac, "Channel occupancy times and handoff rate for mobile computing and PCS networks," IEEE Trans. on Computers, vol. 47, pp. 679-692, June 1998.

[9] Y.-R. Huang, Y.-B. Lin, and J. M. Ho, "Performance analysis for voice/data integration on a finite mobile systems," IEEE Trans. on Vehic. Tech., vol. 49, pp. 367-378, Feb. 2000.

[10] W. Li and X. Chao, "Modelling and performance evaluation of a cellular mobile network," IEEE/ACM Trans. on Networking, vol. 12, pp. 131-145, Feb. 2004.

[11] S. Tang and W. Li, "Performance analysis of a channel allocation scheme for multi-service mobile cellular networks," Int. J. of Communication Systems (IJCS), vol. 20, pp. 177-205, Feb. 2007.

[12] S. Tang and W. Li, "An adaptive bandwidth allocation scheme with preemptive priority for integrated voice/data mobile networks," IEEE Trans. on Wireless Communications, vol. 5, pp. 2874-2886, Oct. 2006. 